\title{
Influence of the mechanical fatigue progress on the magnetic properties of electrical steel sheets
}

\author{
Jan Karthaus ${ }^{1}$, Simon SteentJes ${ }^{1}$, Daniel GröBel ${ }^{2}$, KOLJa ANdReas ${ }^{2}$, \\ MARION MERKLEIN ${ }^{2}$, KAY HAMEYER ${ }^{1}$ \\ ${ }^{1}$ RWTH Aachen University, Institute of Electrical Machines \\ Schinkelstr. 4, 52062 Aachen, Germany \\ e-mail:jan.karthaus@iem.rwth-aachen.de \\ ${ }^{2}$ FAU Erlangen-Nürnberg, Institute of Manufacturing Technology \\ Egerlandstr. 11-13, 91058 Erlangen, Germany
}

(Received: 23.09.2016, revised: 18.01.2017)

\begin{abstract}
The purpose of this paper is to study the variation of the magnetic properties of non-oriented electrical steel sheets with the fatigue state during cyclic mechanical loading. The obtained results are central to the design of variable drives such as traction drives in electric vehicles in which varying mechanical loads, e.g. in the rotor core (centrifugal forces), alter the magnetic properties. Specimens of non-oriented electrical steel are subject to a cyclically varying mechanical tensile stress with different stress amplitudes and number of cycles. The specimens are characterised magnetically at different fatigue states for different magnetic flux densities and magnetising frequencies. The measurements show a variation in magnetic properties depending on the number of cycles and stress magnitude which can be explained by changes in the material structure due to a beginning mechanical fatigue process. The studied effect is critical for the estimation of the impact of mechanical material fatigue on the operational behaviour of electrical machines. Particularly in electrical machines with a higher speed where the rotor is stressed by high centrifugal forces, material fatigue occurs and can lead to deterioration of the rotor's stack lamination.
\end{abstract}

Key words: Cyclic mechanical stress, electrical machines, electrical steel sheets, magnetic properties, material fatigue

\section{Introduction}

The effect of static mechanical stresses is in the focus of recent research. Due to the Villari effect, magnetic hysteresis curves of electrical steel sheets are sheared while loaded by mechanical stress [1]. The shear of the hysteresis curves leads to a higher energy demand and thus to increased iron losses [2]. Due to a variation in the material lattice caused by mechanical stress, the magnetic properties alter. These effects can be observed for mechanical stress within the elastic and plastic region $[1,3]$. 
In electrical machines, mechanical stress appears with static and periodic or cyclic behaviour. Particularly the consequences of static mechanical stress on the iron losses of electrical machines are in the focus of current research [4] as well as the modelling of the magnetomechanical effect [5].

In contrast to the effect of static mechanical stress, the influence of cyclic mechanical stress on the magnetic properties of electrical steel sheet is less studied. Cyclic mechanical stress is caused by a fluctuation of centrifugal forces which are dependent on the speed of the rotor. Furthermore, periodic electromagnetic forces on the rotor surface or stator teeth are the reason for cyclic mechanical stress. Electrical steel packages fatigue mechanically dependent on the number of cycles and the magnitude of the stress. Material fatigue deteriorates the structure of a material starting by a generation of dislocations which then creates micro cracks. At the final state, a dominant crack occurs that leads to total material failure. Fatigue appears already for cyclic mechanical stress amplitudes below yield strength and is dependent on the number of cycles and load amplitude. The fatigue behaviour is also influenced by the geometry of the studied material. In the area around notches such as corners, exaggerated mechanical stresses are induced and lead to a faster material fatigue degradation. Due to the strong interdependence between the material's microstructure and the magnetic properties, fatigue causes a persistent variation in the magnetic properties which is reported in [6-8].

During operational life of constructions or machines, the cyclic mechanical loading of steel parts can lead to an accumulation of fatigue damage. In order to avoid that the fatigue damage process ends in a sudden fracture or significant change in the machine's operating characterristics due to alterations of magnetic properties, it is vital to assess the material degradation. Particularly, this effect can influence the magnetic flux path or the iron losses after a certain time of operation in rotating electrical machines.

In this paper, the influence of the mechanical fatigue progress on the extrinsic magnetic properties (coercivity, remanence, specific losses) of a non-oriented electrical steel sheet for the uniaxial case is studied. At several interruptions of the cyclic load, the magnetic properties are measured using a standard single-sheet-tester (SST). The results show a variation in the magnetic properties depending on the number of cycles and the level of mechanical stress.

\section{Cyclic stress}

Cyclic mechanical loading of a specimen causes material fatigue that can result in failure at stress levels below the static tensile strength of the material [9]. This material degradation process is caused by a movement and agglomeration of dislocations followed by nucleation and growth of micro cracks. Micro cracks emanate from stress sensitive notches on the surface of the specimen or from inclusions in the material volume which are both a result of the manufacturing process [10]. With ongoing stress exposure, the cracks proceed to enlarge due to repeated plastic flow at the crack edges whereby the crack front evolves perpendicular to the direction of the principal stress vector. Finally, the crack sizes reach critical values that lead to 
a rapid weakening of the material and ultimately to an instable residual fracture of the specimen [9].

Table 1. Mechanical properties of M250-35A

\begin{tabular}{l|c|c}
\hline \multicolumn{1}{c|}{ Description } & Symbol & Value \\
\hline Yield Strength & $\sigma_{y}$ & $361 \mathrm{MPa}$ \\
\hline Young's modulus & $E$ & $210 \mathrm{GPa}$ \\
\hline Tensile strength & $\sigma_{T S}$ & $471 \mathrm{MPa}$ \\
\hline
\end{tabular}

A widely-used method to characterise the fatigue behaviour of steels is the Wöhler $(s-N)$ approach which relates the number of cycles to failure to the fatigue stress [11]. In order to determine the fatigue strength for different zones of load cycles, specimens are stressed with constant stress or strain values until failure occurs. Typical values of cycle numbers range from $N=[1,104]$ for low cycle fatigue, $N=[104,107]$ for high cycle fatigue and $N>107$ for endurance fatigue [11].

\subsection{Investigated material}

To analyse the effect of cyclic stress on the magnetic properties of electrical steel sheet, sheets of type M250-35A cut by a CO2-laser are used. Due to the used cutting method, material deterioration at the cutting edges can be expected. In this study, this effect is neglected because each specimen has the same amount of material deterioration due to cutting. Examining the determined stress-strain curve of the studied material, the mechanical data of the material is collected in Table 1. Only specimens in rolling direction are studied. The shape of the specimens to apply cyclic loads is shown in Fig. 1 b.

\subsection{Cyclic load}

To analyse the variation of the magnetic properties due to fatigue evolution, specimens are loaded at different stress levels and different numbers of cycles using the hydraulic testing machine HA250 (Zwick). The hydraulic driven machine enables to test flat specimens by means of an application of cyclic uniaxial loads with a frequency of $20 \mathrm{~Hz}$. The specimen design is orientated on the recommendations of the norm [12]. An influence of the testing frequency on the fatigue behaviour or the magnetic properties will be investigated in further studies. However, adjustments have to be made in order to fulfil the requirements of the clamping conditions and the magnetic measurement system (Fig. 1a). With the objective to provide constant conditions for every parameter setting, the specimens are not tested as single sheets, but as packages of six specimens. To ensure the same load for every sheet, the machining of the final geometry is conducted in packages of six sheets as well.

For this study, a cyclic tensile load following a sinusoidal-shaped curve is used. To avoid compressive stresses on the specimen, the pre-load has a minimum value of $\sigma_{\min }=1.6 \mathrm{MPa}$. In contrast to conventional fatigue tests, no cracking of the specimen is intended here. Hence, the maximum stress is determined to $70 \%$ of the static tensile strength. To provide sufficient data points, three stress levels are chosen which amount to $0.23 \sigma_{y}(83 \mathrm{MPa}), 0.46 \sigma_{y}(165 \mathrm{MPa})$ 
and $0.69 \sigma_{y}(249 \mathrm{MPa})$. Furthermore, to enable insights into the development of the magnetic properties in respect to the cycle number with sufficient resolution, six different values ranging from 10000 to 500000 cycles are studied for stress levels of $83 \mathrm{MPa}$ and $165 \mathrm{MPa}$. For a load of $249 \mathrm{MPa}$, only cycle numbers of up to 100000 are utilized since cracking of the specimen is expected for higher values.

Table 2. Stress levels and corresponding number of cycles performed

\begin{tabular}{l|c}
\hline Stress level $\sigma_{\max }$ & Number of cycles $N$ \\
\hline $83 \mathrm{MPa}$ & $500000 / 250000 / 100000 / 50000 / 25000 / 10000$ \\
\hline $165 \mathrm{MPa}$ & $500000 / 250000 / 100000 / 50000 / 25000 / 10000$ \\
\hline $249 \mathrm{MPa}$ & $100000 / 50000 / 25000 / 10000$ \\
\hline
\end{tabular}
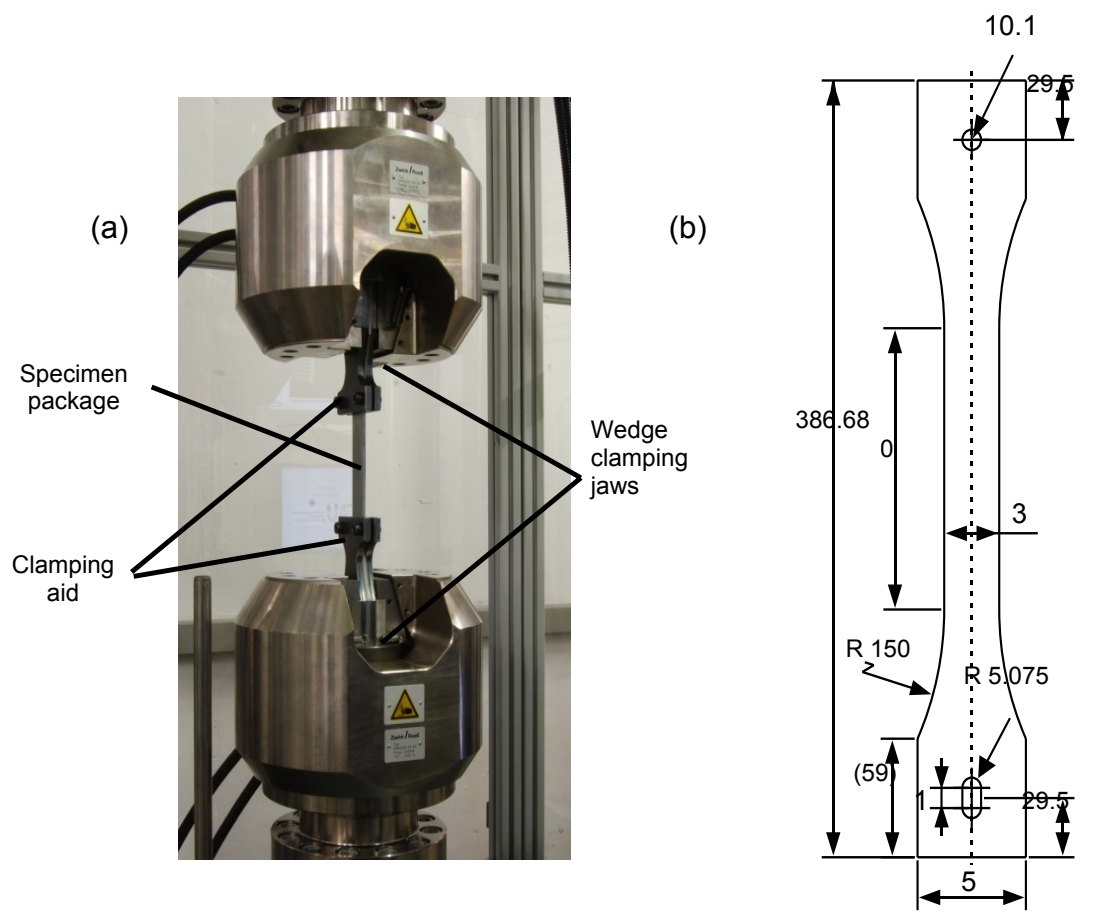

Fig. 1. Experimental setup (a) and geometric properties of the specimen (b)

A detailed overview of the experimental design is depicted in Table 2.

\section{Magnetic measurements}

\subsection{Experimental setup}

Four specimens of each load state (Table 2) are stressed in a cyclic way. Due to the required sample width of $120 \mathrm{~mm}$ of the magnetic testing setup, which is described below, the 
central parts of four specimens that were loaded mechanically are cut, merged and fixed by a non-magnetic adhesive tape to a sample with a width of $120 \mathrm{~mm}$ to fit into the used SST (Fig. 2).

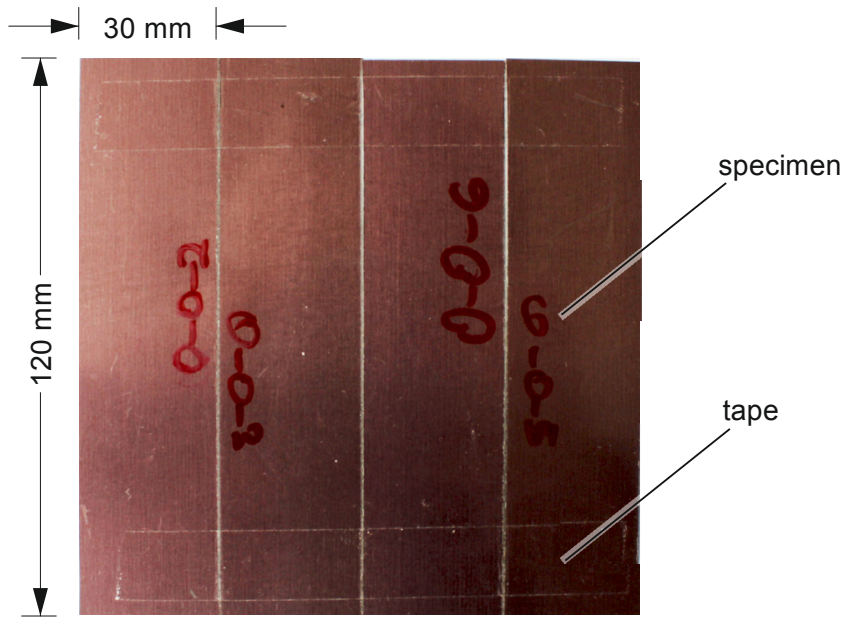

Fig. 2. Merged specimen with tape

For each stress level and number of cycles, one of the expanded specimens is measured magnetically by a standard SST constructed for samples with a width and length of $120 \mathrm{~mm}$. The usage of the expanded specimens reduces the discrepancy of the results of the fatigue experiments (Fig. 6).

The SST is incorporated into a computer-aided setup in accordance with the international standard [13]. The samples are characterised using controlled sinusoidal magnetic flux density with a form factor error of less than $1 \%$ in the frequency range from quasi-static to $1 \mathrm{kHz}$.

\subsection{Results}

Regarding the variations in the hysteresis curves depending on the fatigue state under a constant stress level (Fig. 3), a shear in the hysteresis curves which results in a small increase of coercivity and a decrease of remanence polarisation is observed. It is apparent that for an increasing number of load cycles, the amount of magnetic field strength to reach the same magnetic polarisation increases as well. This is valid both for low and high magnetising frequencies (Fig. 3).

An influence of the stress level is also apparent for a constant number of load cycles (Fig. 4). While for the stress levels up to $165 \mathrm{MPa}$, the shear of the hysteresis curves is insignificantly small, the variation for higher stress levels is obvious. This behaviour can be explained by a larger variation in the microstructure at higher mechanical stresses.

Fig. 5 depicts the trend of the different magnetic properties such as coercive field, remanence polarisation and specific losses for various stress levels depending on the number of cycles for two operating points $\left(f=50 \mathrm{~Hz}, J_{\max }=1.5 \mathrm{~T}\right.$ and $\left.f=400 \mathrm{~Hz}, J_{\max }=1.5 \mathrm{~T}\right)$. 
The behaviour of the magnetic properties can be separated into three stages which can also be found in the literature $[14,16]$ :

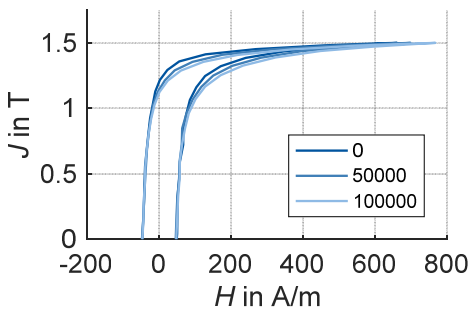

(a) $f=50 \mathrm{~Hz}$ and $J_{\max }=1.5 \mathrm{~T}$

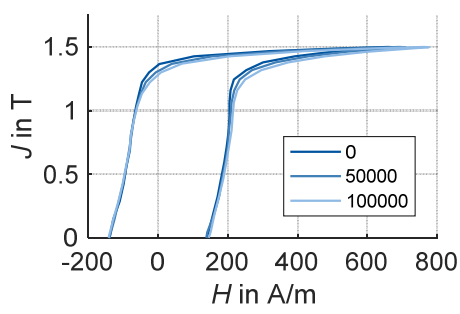

(b) $f=400 \mathrm{~Hz}$ and $J_{\max }=1.5 \mathrm{~T}$

Fig. 3. Hysteresis curves of stressed samples depending on the number of load cycles $N$ for $\sigma_{\max }=249 \mathrm{MPa}$

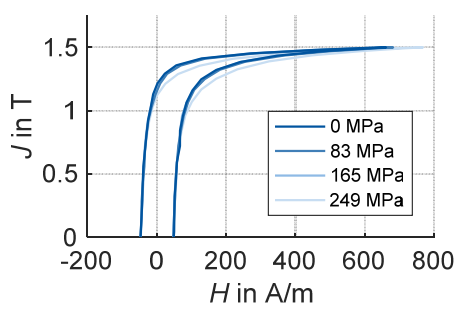

(a) $f=50 \mathrm{~Hz}$ and $J_{\max }=1.5 \mathrm{~T}$

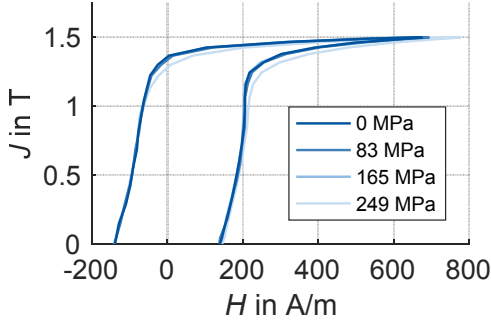

(b) $f=400 \mathrm{~Hz}$ and $J_{\max }=1.5 \mathrm{~T}$

Fig. 4. Hysteresis curves of stressed samples depending on the mechanical stress level $\sigma_{\max }$ for $N=100000$

During the first percent of the fatigue life-time, the dislocation density increases which is indicated by an increase of coercivity, i.e. fatigue hardening occurs. This behaviour is observable for all studied stress stages. The occurrence of fatigue hardening is typical for heattreated materials [14]. At the same time, the remanence polarisation is decreasing. At this stage, an increase of losses, i.e. increase of coercivity and decrease of remanence, is apparent, seemingly due to the negative effect of an increase of the dislocation density inside the material.

In the next stage, between 100000 and 250000 cycles, the magnetic properties remained almost unchanged for stress levels of $83 \mathrm{MPa}$ and $165 \mathrm{MPa}$. Because of the more progressed fatigue process for $249 \mathrm{MPa}$, this behaviour is not observable for this stress level. At this stage, the number of dislocations stays almost constant which results in the constant magnetic properties. The existing dislocations tend to move to the surface of the specimen [14].

In the last stage, the magnetic properties are altered significantly. Due to the fatigue progress, micro cracks are formed and propagate within the material before creating a final crack and failure of the material. 


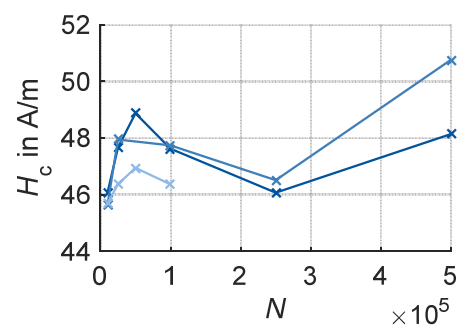

(a) Coercive field for $f=50 \mathrm{~Hz}$ and $J_{\max }=1.5 \mathrm{~T}$

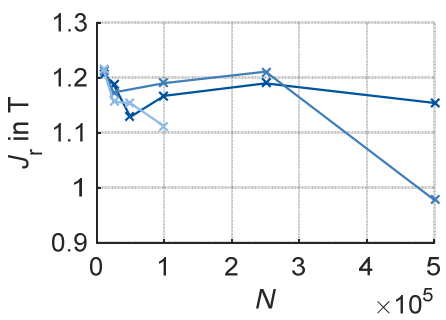

(c) Remanence polarisation for $f=50 \mathrm{~Hz}$ and $J_{\max }=1.5 \mathrm{~T}$

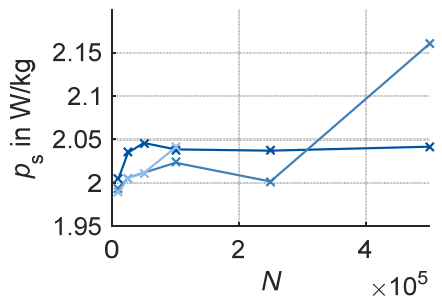

(e) Specific losses for $f=50 \mathrm{~Hz}$ and $J_{\max }=1.5 \mathrm{~T}$

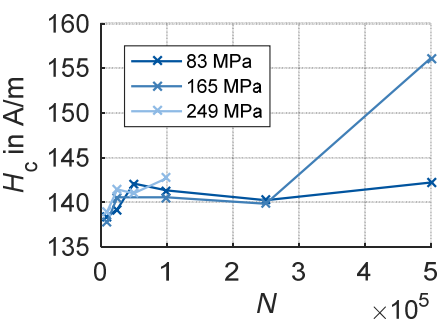

(b) Coercive field for $f=400 \mathrm{~Hz}$ and $J_{\max }=1.5 \mathrm{~T}$

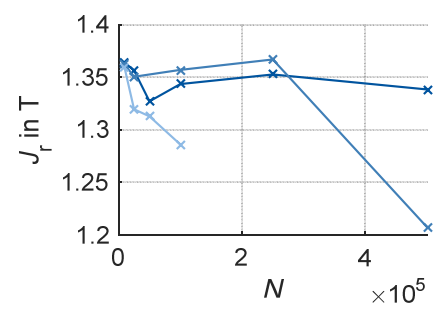

(d) Remanence polarisation for $f=400 \mathrm{~Hz}$ and $J_{\max }=1.5 \mathrm{~T}$

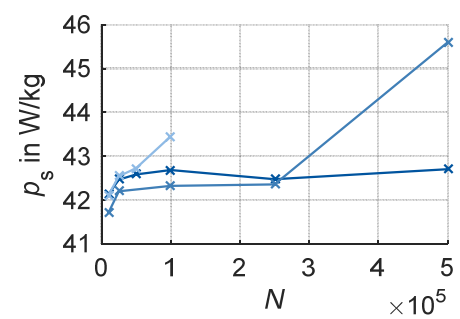

(f) Specific losses for $f=400 \mathrm{~Hz}$ and $J_{\max }=1.5 \mathrm{~T}$

Fig. 5. Coercive field, remanence polarisation and specific losses at different stress levels depending on the number of load cycles at various operating points

The magnetic properties are strongly depending on the crack size [15]. It is apparent that for a higher stress level, the final fatigue state is reached for a smaller number of cycles while for a low stress level, the final fatigue stage is not reached yet, observing the small variation in magnetic properties for $83 \mathrm{MPa}$.

Although the variation of the magnetic properties is only a few percent, a clear trend is observable validated by different magnetising frequencies. Compared to the influence of static stress on the magnetic properties, the influence of cyclic stress is seemingly low.

Fig. 6 depicts the mean values and corresponding standard deviations for each measurement of the six original specimens for one stress level and number of cycles at one magnetic operating point. Due to the fact that the mechanical cyclic load is subject to statistical uncertainties, a wide variation for each measurement can be observed. Another reason is the small width of the specimen $(30 \mathrm{~mm})$ which is crucial for the SST which requires samples 
with a width of $120 \mathrm{~mm}$. Nevertheless, a clear trend is observable which is validated by the more exact measurements with the merged sample of $120 \mathrm{~mm}$ as well.

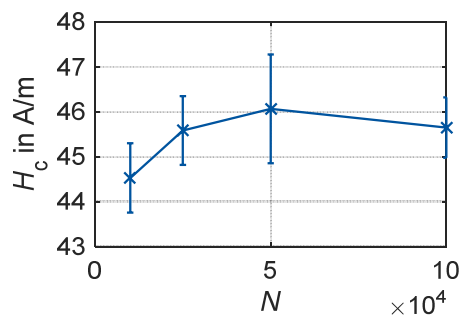

(a) Coercivity

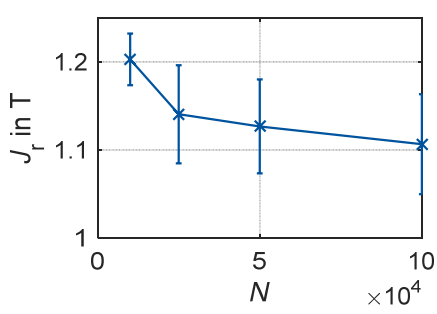

(b) Remanence polarisation

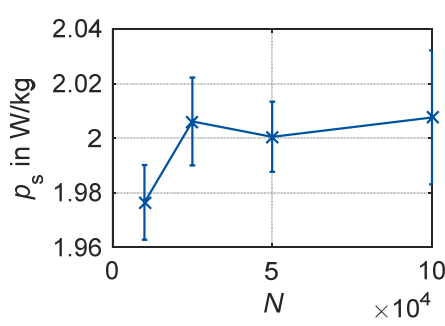

(c) Specific losses

Fig. 6. Mean value (x) and standard deviation of the measurement of the magnetic properties for original samples for $J=1.5 \mathrm{~T}, f=50 \mathrm{~Hz}$ and $\sigma_{\max }=249 \mathrm{MPa}$

According to this study, the fatigue-depending behaviour of the magnetic properties of electrical steel sheet cannot be neglected. In particular, in the first percentage of the fatigue progress to 100000 load cycles, a large variation in magnetic properties is observed. The effect on the hysteresis curves and the iron losses can lead to different machine behaviour after a certain time of operation, depending on the cyclic load which affects the stack laminations. For example, fatigue can be induced by centrifugal forces that cause mechanical stress within the stack lamination or by electromagnetic forces causing mechanical stress in stator teeth and rotor edge. Regarding the rotor of an electrical machine with higher speeds (e.g. traction drives of electric vehicles), mechanical tensile stresses up to the yield strength of the steel sheet material, e.g. $320 \mathrm{MPa}$ can occur. Examining the results of this study, the magnetic properties of the areas with high mechanical stresses are altered more intensively. Even though rotors of electrical machines are not loaded with a large number of cycles which initialise cracks, there is a large variation in magnetic properties for low number of cycles.

As a result of this study, it is evidenced that the influence on the magnetic properties of non-oriented electrical steel sheet is depending on the number of load cycles and stress level. Since the fatigue process is more intensive for mechanical stress levels closer to the yield strength of the material, i.e. the final failure occurs after a smaller number of cycles, the deterioration of magnetic properties is larger in the area of high mechanical stresses. For example, in permanent-magnet synchronous machines with a higher speed, the rotor stiffness 
is influenced by the position and geometry of the magnet pockets. Operating the machine with cyclic speeds, thin bridges and notches lead to high mechanical stresses and material fatigue in the materials. As a consequence, the magnetic properties alter due to static stress and cyclic stress. Furthermore, mechanical fatigue can produce changes in the microstructure which lead to micro cracks and component failure. Additional influences such as the mechanical stress due to electromagnetic forces or the mechanical stress induced by cutting and punching of the electrical steel sheets can lead to an accelerated fatigue progress.

\section{Conclusions}

In this paper, the effect of cyclic stress on the magnetic properties of electrical steel sheet is studied. The relation between magnetic properties and fatigue state is presented. Particularly, for a small number of load cycles, the variation in the magnetic properties is higher when compared to a high number of cycles. The stress level also shows an influence on the intensity of variation at larger mechanical load.

In this study, three typical fatigue states have been identified and explained by changes in the microstructure and occurrence of micro cracks within the electrical steel sheet. The results of fatigue-depending magnetic measurements of different steel materials of other authors support the observed results $[14,16]$. Compared to the influence of static mechanical stress, the influence of cyclic stress is low. The influence of the superposition of cyclic and static stress on the magnetic properties has to be analysed.

The study will be continued for a smaller number of cycles $(N<10000)$ because the results show a large variation in magnetic properties in the first fatigue state. The used measurement approach, combining cyclically-loaded specimens and a standardised magnetic SST lead to appropriate measurement results which is comparable to other measurement techniques such as presented in [17].

This study is done by rectangular specimens loaded in a uniaxial way and magnetic measurements collinear to the rolling direction. Neither the influence multiaxial loadings nor rotating magnetic fields are covered within this study. Further investigations with different measurement equipment such as a rotating SST and notched specimens will be performed. For additional studies, a higher number of specimens will be used to provide trends with a higher statistical value.

Depending on the state of an electrical machine's operational life and its cyclic mechanical loads, the magnetic properties are altered in a noticeable way, i.e. the iron losses and coercive field are increased while the remanence polarisation is decreased. Particularly for a number of cycles up to 100000 , the largest variation in properties is overserved. For example, the total iron losses increase about $4 \%$ when compared to the initial state at $400 \mathrm{~Hz}$. For the operational life of electrical machines, it can be assumed that the overall efficiency and the machine's behaviour due to a variation in the magnetic flux path may be changed. 


\section{Acknowledgements}

This work is originated as part of the research training group GRK 1856 by the Deutsche Forschungsgesellschaft (DFG)

\section{References}

[1] Leuning N., Steentjes S., Schulte M., Bleck W., Hameyer K., Effect of elastic and plastic tensile mechanical loading on the magnetic properties of NGO electrical steel, Journal of Magnetism and Magnetic Materials, vol. 417, pp. $42-48$ (2016).

[2] Permiakov V., Dupré L., Pulnikov A., Melkebeek J., Loss separation and parameters for hysteresis modelling under compressive and tensile stresses, Journal of Magnetism and Magnetic Materials, vol. 272-276, pp. E553-E554 (2004).

[3] Hug E., Evolution of the magnetic domain structure of oriented 3\% SiFe sheets with plastic strains, Journal of Materials Science, vol. 30, no. 17, pp. 4417-4424 (1995).

[4] Singh D., Rasilo P., Martin F., Belahcen A., Arkkio A., Effect of Mechanical Stress on Excess Loss of Electrical Steel Sheets, IEEE Transactions on Magnetics, vol. 51, no. 11, pp. 1-4 (2015).

[5] Vanoost D., Steentjes S., Peuteman J., Gielen G., de Gersem H., Pissoort D., Hameyer K., Magnetic hysteresis at the domain scale of a multi-scale material model for magneto-elastic behaviour, Journal of Magnetism and Magnetic Materials, vol. 414, pp. 168-179 (2016).

[6] Devine M.K., Jiles D.C., Hariharan S., Effects of cyclic stress on the magnetic hysteresis parameters of polycrystalline iron, Journal of Magnetism and Magnetic Materials, vol. 104-107, pp. $377-$ 378 (1992).

[7] Bozorth R.M., Ferromagnetism, IEEE Press; Wiley-Interscience (1993).

[8] Gilanyi A., Morishita K., Sukegawa T., Uesaka M., Miya K., Magnetic nondestructive evaluation of fatigue damage of ferromagnetic steels for nuclear fusion energy systems, Fusion Engineering and Design, vol. 42, no. 1-4, pp. 485-491 (1998).

[9] Radaj D., Ermüdungsfestigkeit: Grundlagen für Leichtbau, Maschinen- und Stahlbau, Springer Verlag (1995).

[10] Murakami Y., Kodama S., Konuma S., Quantitative evaluation of effects of non-metallic inclusions on fatigue strength of high strength steels. I: Basic fatigue mechanism and evaluation of correlation between the fatigue fracture stress and the size and location of non-metallic inclusions, International Journal of Fatigue, vol. 11, no. 5, pp. 291-298 (1989).

[11] Testing of materials; fatigue test (Woehlertest); definitions, symbols, execution, evaluation, DIN 50100:1978-02 (1978).

[12] Metallic materials - Tensile testing - Part 1: Method of test at room temperature (ISO 68921:2009), DIN EN ISO 6892-1:2009-12 (2009).

[13] Magnetic materials - Part 3: Methods of measurement of the magnetic properties of electrical steel strip and sheet by means of a single sheet tester (IEC 60404-3:1992 + A1:2002 + A2:2009), DIN IEC 60404-3:2010-05 (2010).

[14] Lo C.C., Tang F., Jiles D.C., Biner S.B., Evaluation of fatigue damage using a magnetic measurement technique, IEEE Transactions on Magnetics, vol. 35, no. 5, pp. 3977-3979 (1999).

[15] Bi Y., Jiles D.C., Dependence of Magnetic Properties on Crack Size in Steels, IEEE Transactions on Magnetics, vol. 34, no. 4, pp. 2021-2023 (1998).

[16] Devine M.K., Kaminski D.A., Sipahi L.B., Jiles D.C., Detection of Fatigue in Structural Steels by Magnetic Property Measurements, Journal of Materials Engineering and Performance, vol. 1, no. 2, pp. 249-253 (1992).

[17] Jiles D.C., Hariharan S., Devine M.K., Magnescope: a portable magnetic inspection system for evaluation of steel structures and components, IEEE Transactions on Magnetics, vol. 26, no. 5, pp. 2577-2579 (1990). 